\title{
On the Accuracy of EGM2008 with Measured Data Based on Strap-down Airborne Gravimetry
}

\author{
Shaoliang $\mathrm{Li}^{1, \mathrm{a}}$, Meiping $\mathrm{Wu}^{1, \mathrm{~b}}$, Shaokun $\mathrm{Cai}^{1, \mathrm{c}}$ \\ ${ }^{1}$ College of Mechatronics and Automation, National University of Defense Technology, Changsha \\ 410073, China

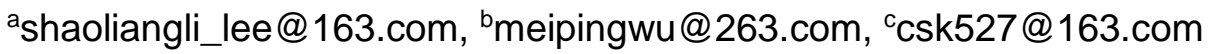

Keywords: strap-down airborne gravimetry, EGM2008, accuracy.

\begin{abstract}
Gravity survey is an important research topic in geophysics and geodynamics. This paper analyzes the accuracy of EGM2008, the newest Earth gravitational model, in two areas in Shandong Province of China. By comparing the gravity anomaly data from the model with those obtained by a strap-down airborne gravimeter called SGA-WZ, it shows that error accuracy between the gravity anomaly calculated from EGM2008 and the measured data in these two areas is within $2.16 \mathrm{mGal}$.
\end{abstract}

\section{Introduction}

The Earth's gravity field is a fundamental physical field. It has connections with all the physical events on earth and in near space and provides the basic information to support research on many subjects, such as geodesy, geophysics, geodynamics and resource exploration. The Earth Gravitational Model, EGM2008, released by Goddard Space Flight Center of National Aeronautics and Space Administration (NASA/GFSC), is a spherical harmonic model of the Earth's gravitational potential, developed by using a combination of terrestrial gravity data and gravity data obtained by satellite gravity data. The order of the model can reach 2159, and the parameters which could expand it to 2190 order are provided [1].

Airborne gravimetry is an effective method to collect the homogeneous distribution, precise and large area information about the Earth's gravity. A strap-down airborne gravimeter has many advantages of being simple structure, small size, light weight, low cost, low power consumption. Based on airborne gravimetry technology, several flight tests were carried out in Shandong Province of China with the strap-down airborne gravimeter, called SGA-WZ, developed by the Laboratory of Inertial Technology of the National University of Defense Technology [2-4]. This paper analyzes the error accuracy of the gravity anomaly calculated from EGM2008 by using measured data from SGA-WZ.

\section{Basic Principles}

Airborne Gravimetry Technology. According to Newton's Second Law, the equations of particle dynamics with respect to inertial frame (i-frame) could be written as

$$
f^{i}=\ddot{r}^{i}-g^{i}
$$

where $\ddot{r}^{i}$ is the acceleration of the particle dynamics in i-frame, $g^{i}$ and $f^{i}$ are the true gravitational acceleration and specific force sensed by the accelerometers respectively. Thus, rearranging the equation yields

$$
g^{i}=\ddot{r}^{i}-f^{i}
$$

Equation (2) is the basic principle of airborne gravimetry.

Specific force equation established in the local geographic frame (n-frame) is:

$$
\dot{v}_{e}^{n}=C_{b}^{n} f^{b}-\left(2 \omega_{i e}^{n}+\omega_{e n}^{n}\right) \times v_{e}^{n}+g^{n}
$$


where $\dot{v}_{e}^{n}$ is the acceleration, $v_{e}^{n}$ is the velocity with respect to earth surface in the n-frame, $f^{b}$ is the specific force sensed by accelerometers in the b-frame, $C_{b}^{n}$ is the transformation matrix from the platform's b-frame to the n-frame which is computed by the attitude angles (roll, pitch and yaw). $g^{n}$ is the actual gravity vector. $\omega_{i e}^{n}$ is the Earth's rotation rate in the n-frame, $\omega_{e n}^{n}$ is the turn rate of n-frame with respect to Earth in the n-frame.

The gravity disturbance vector of the Earth is calculated by Equation (4) [5]:

$\delta g^{n}=g^{n}-\gamma^{n}$

Where $\delta g^{n}$ is gravity disturbance, and $\gamma^{n}$ is the normal gravity vector in the $\mathrm{n}$-frame.

By using equation (3) and (4), we can get the basic equation of strap-down airborne gravimetry:

$\delta g^{n}=\dot{v}_{e}^{n}-C_{b}^{n} f^{b}+\left(2 \omega_{i e}^{n}+\omega_{e n}^{n}\right) \times v_{e}^{n}-\gamma^{n}$

Equation (5) is the mathematics model to implement the vector gravimetry, and it can be expanded to three components:

$$
\left\{\begin{array}{l}
\delta g_{E}=\dot{v}_{E}-f_{E}+\left(\frac{v_{E}}{R_{N}+h}+2 \omega \cos \varphi\right)\left(v_{U}-v_{N} \tan \varphi\right) \\
\delta g_{N}=\dot{v}_{N}-f_{N}+\left(\frac{v_{E}}{R_{N}+h}+2 \omega \cos \varphi\right) \tan \varphi \cdot v_{E}+\frac{v_{N} v_{U}}{R_{M}+h} \\
\delta g_{U}=\dot{v}_{U}-f_{U}-\left[\left(\frac{v_{E}}{R_{N}+h}+2 \omega \cos \varphi\right) \cdot v_{E}+\frac{v_{N}^{2}}{R_{M}+h}\right]-\gamma_{U}
\end{array}\right.
$$

where $\dot{v}_{N} \quad \dot{v}_{E} \quad \dot{v}_{U}$ are the north, east and down acceleration of the vehicle with respect to the Earth in the n-frame, respectively, $v_{N}, v_{E}$ and $v_{U}$ are the north, east and down velocity of the vehicle with respect to the Earth in the n-frame, respectively, $f_{N} f_{E}$ and $f_{U}$ are the north, east and down specific force, respectively, $R_{M}$ and $R_{N}$ are the meridian and prime vertical radii of curvature, respectively, $L$ and $h$ are the latitude and height, respectively, $\omega$ is the earth rotation rate, and $\gamma_{U}$ is the normal gravity.

This paper just considers about the scalar information of gravity disturbance, i.e. the vertical component of gravity disturbance.

Basic Equations of Gravity Field. Earth's gravitational potential, $T$, in spherical harmonics can be expressed as [6]:

$$
T=\frac{f M}{\rho} \sum_{n=2}^{N}\left[\left(\frac{R}{\rho}\right)^{n} \sum_{m=0}^{n}\left(\bar{C}_{n m} \cos m \lambda+\bar{S}_{n m} \sin m \lambda\right) \bar{P}_{n m}(\cos \theta)\right]
$$

where $f M=3.986004415 \times 1014 \mathrm{~m}^{3} \mathrm{~s}^{-2}$ is the product of Newton's gravitational constant and Earth's mass, $R=6.3781363 \times 10^{6} \mathrm{~m}$ is the mean radius of Earth, $\rho, \theta$ and $\lambda$ are the radial distance, colatitude, and longitude of the point, respectively, $\bar{P}_{n m}$ are the normalized Legendre polynomials, $\bar{C}_{n m}$ and $\bar{S}_{n m}$ are the normalized spherical harmonic coefficients of degree $n$ and order $m$ respectively.

Under the condition of sphere approximate, the gravity disturbance ( or so-called gravity anomaly) on the surface has the relationship with gravitational potential as [7]:

$$
\Delta g=-\frac{\partial T_{R}}{\partial \rho}+\frac{2}{\rho} T_{R}
$$

Where $T_{R}$ is the gravity disturbance on the surface. Substitute Equation (7) into equation (8):

$$
\Delta g=\frac{f M}{R^{2}} \sum_{n=2}^{N}\left[(n-1) \sum_{m=0}^{n}\left(\bar{C}_{n m} \cos m \lambda+\bar{S}_{n m} \sin m \lambda\right) \bar{P}_{n m}(\cos \theta)\right]
$$




\section{Test Results and Data Analysis}

Description of the Flight Tests. The strap-down airborne gravimeter, SGZ-WZ, is the first gravimeter based on the strap-down inertial navigation system that have ever made in China. This system constitutes of a high-performance strap-down inertial navigation system (SINS), a GNSS receiver, an anti-vibration system, a data logger and post-processing software. The major advantage of this system is its reliability and robustness in operations. A photograph of the system is shown in Figure 1.

The flight tests were carried out in 2 areas in Shandong Province of China. SGA-WZ system was onboard a Cessna 208 aircraft. The average altitude of the flights was $400 \mathrm{~m}$. And the flight lines were both along with the direction of parallel, covering around $1.36^{\circ}$ in longitude. Figure 2 shows Cessna 208 fixed-wing aircraft.

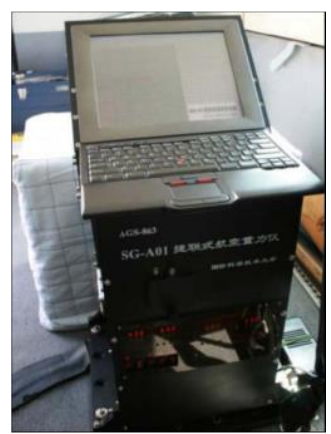

Fig. 1 SGA-WZ system

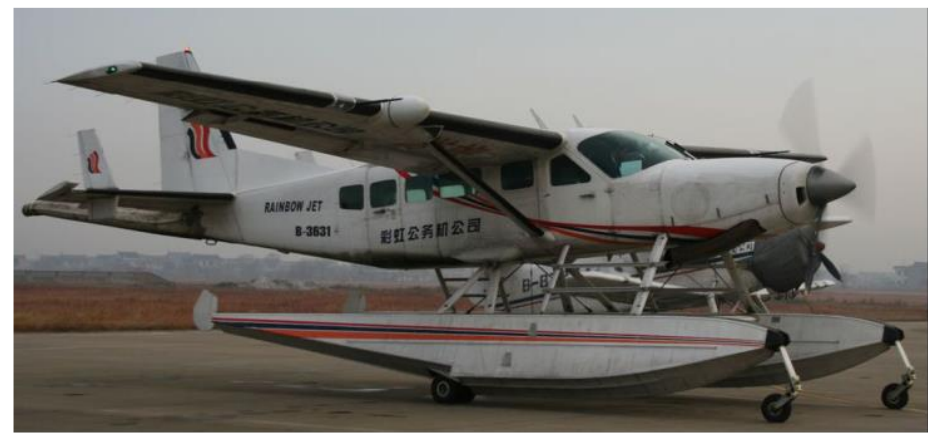

Fig. 2 Cessna 208 fixed-wing aircraft

Data Processing and Analysis. The data sources used in this paper is $2.5^{\prime} \times 2.5^{\prime}$ grid EGM2008 Earth Gravitational Model with the order of 2159. It offers $\left(\bar{C}_{n m}, \bar{S}_{n m}\right)$ of points in accordance with their coordinates. With the help of Equation (9), gravity anomaly data corresponding to the test areas was worked out. However, the number of model data points is much less than the measured data points. Then, this model data is processed by using Line Interpolation method. The interpolated model data and the measured data in the two areas were showed in Figure 3.1 and 4.1, respectively, the errors of the two sources of data in the two areas are showed in Figure 3.2 and 4.2, respectively. Table 1 shows the statistical characteristics of the errors of the two tests.

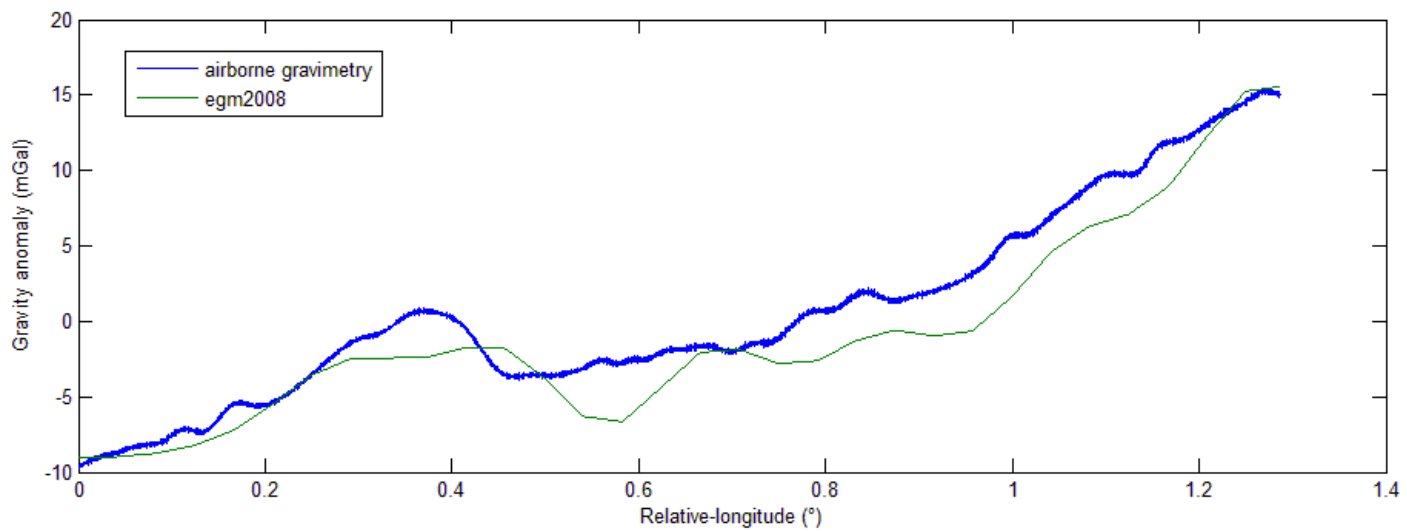

Fig. 3.1 Comparison of gravity anomaly in the first test

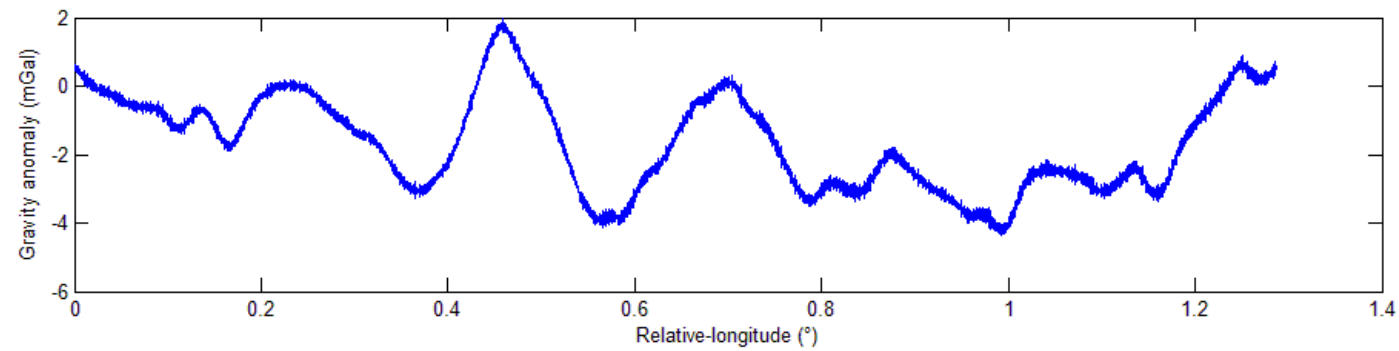

Fig. 3.2 Errors of the two sources of data in the first test 


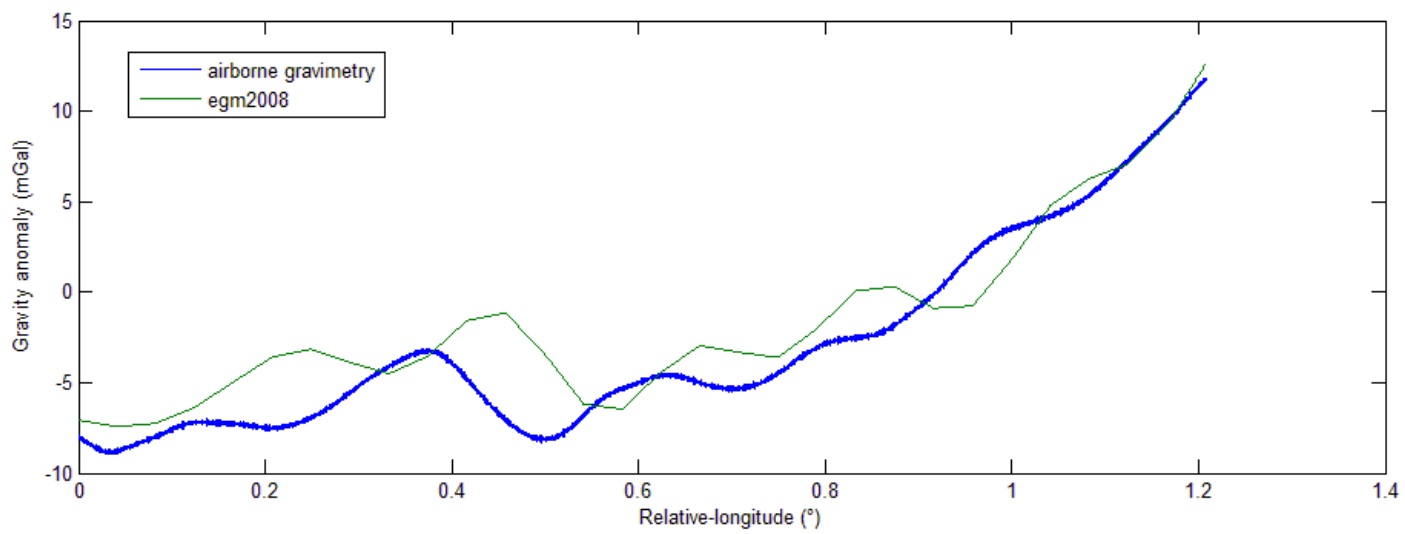

Fig. 4.1 Comparison of gravity anomaly in the second test

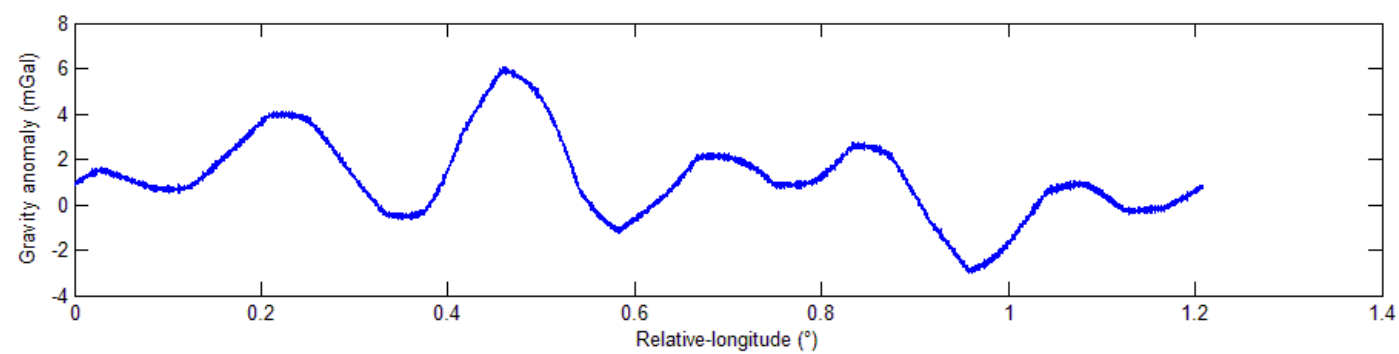

Fig. 4.2 Errors of the two sources of data in the second test

Table 1 Statistical characteristics of the errors of the two tests mGal

\begin{tabular}{ccccc}
\hline Numble & Min & Max & Mean & RMS \\
\hline 1 & -4.3389 & 1.9654 & -1.6466 & 2.1599 \\
2 & -3.0243 & 6.0778 & 1.1752 & 2.1535 \\
\hline
\end{tabular}

The accuracy of SGA-WZ is $1 \mathrm{mGal}$ [8], which confirms the reliability of the measured data. Results show that model data has a fluctuate error according to measured data. It also illustrates that in both of the two regions, error precision between the gravity anomaly calculated from GM2008 and the measured data is within $2.16 \mathrm{mGal}$, which can meet the need of precision requirement in electrical, magnetic and chemical exploration.

\section{Summary}

Based on the airborne gravimetry technology, a custom designed strap-down airborne gravimeter, SGA-WZ, developed by the Laboratory of Inertial Technology of the National University of Defense Technology, was used to carry out flight tests in two areas in Shandong Province of China to analyze the performance of the latest Earth gravity model EGM2008 in China. This paper uses Line Interpolation method to process the data calculated from EGM2008, and compares it with the measured gravity anomaly data obtained from SGA-WZ. Results show that the error precision between the gravity anomaly calculated from GM2008 and that from measured data is within $2.16 \mathrm{mGal}$, which can meet the need of precision requirement in electrical, magnetic and chemical exploration.

\section{References}

[1] Pavlis N K, Holmes S A, Kenyon S C, et al. The development and evaluation of the Earth Gravitational Model 2008 (EGM2008)[J]. Journal of Geophysical Research: Solid Earth (1978-2012), 2012, 117(B4).

[2] Zhang, K.D. Research on the Methods of Airborne Gravimetry Based on SINS/DGPS. Ph.D. Dissertation. National University of Defense Technology, Changsha, China, 2007. 
[3] Huang, Y.M.; Olesen, A.V.; Wu, M.P.; Zhang, K.D. SGA-WZ: A new strap-down airborne gravimeter. Sensors 2012, 12, 9336-9348.

[4] Cai, S.K.; Zhang, K.D.; Wu, M.P.; Huang, Y.M. Long-term stability of the SGA-WZ strap-down airborne gravimeter. Sensors 2012, 12, 11091-11099.

[5] Hackney R I, Featherstone W E. Geodetic versus geophysical perspectives of the 'gravity anomaly'[J]. Geophysical Journal International, 2003, 154(1): 35-43.

[6] Pavlis N K, Holmes S A, Kenyon S C, et al. An earth gravitational model to degree 2160: EGM2008[J]. EGU General Assembly, 2008: 13-18.

[7] Rapp R H, Pavlis N K. The development and analysis of geopotential coefficient models to spherical harmonic degree 360[J]. Journal of Geophysical Research: Solid Earth (1978-2012), 1990, 95(B13): 21885-21911.

[8] Cai S K, Wu M P, Zhang K D, et al. The first airborne scalar gravimetry system based on SINS/DGPS in China[J]. Science China Earth Sciences, 2013, 56(12): 2198-2208. 